\title{
RECENT DEVELOPMENT IN UNDERSTANDING BEAM LOSS IN HIGH-INTENSITY SYNCHROTRONS
}

\author{
G. Franchetti* GSI Darmstadt, Germany
}

\section{Abstract}

We report about recent advances in understanding spacecharge dependent beam loss and emittance growth driven by nonlinear dynamics, which allow quantitative predictions for a large number of turns (typically $10^{5}$ or more). In this talk we review the theoretical model of trapping by space charge effects, simulation results and experimental findings obtained at the CERN Proton Synchrotron and the heavy ion synchrotron SIS18 at GSI. The impact of these effects on the beam loss budget/beam loss control for heavy ion beams in the SIS100 synchrotron in the FAIR project will be presented.

\section{INTRODUCTION}

The new physics research frontiers are worldwide requiring the use of high intensity beams provided in dedicated accelerator facilities. In Fig. 1 is shown a summary of the major laboratories in which operation with high intensity beams takes place. For each synchrotron is considered the beam production performance in terms of the space charge detuning $\Delta Q$ jointly to the turns $N$ without major loss in which such a beam is kept in the accelerator. Several accelera-

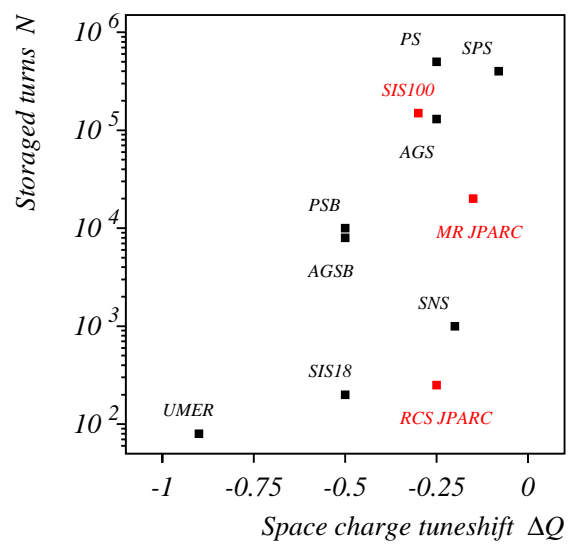

Figure 1: Classification of the high intensity rings with ring under construction marked in red.

tors (black marks) are running with high intensity for short term storage or injection plateau, typically $10^{4}$ turns: SNS [1], AGS Booster at BNL [2] PSB at CERN [3] and SIS18 at GSI [4]. Some rings under construction as the RCS at JPARC [5] will operate also in this regime. Already in the short term storage of high intensity beams, space charge poses important beam control challenges which we shortly address in the next section. In the JPARC project the main

\footnotetext{
*g.franchetti@gsi.de
}

05 Beam Dynamics and Electromagnetic Fields ring (MR) exceeds the $10^{4}$ turns storage time. The AGS at BNL also operates in a similar condition [2], but the injection and accumulation in $750 \mathrm{~ms}$ happens with beam loss of $20 \%$. After injection the beam remains stored for $1.3 \times 10^{5}$ turns. We take the value of $\sim 10^{4}$ turns as somewhat arbitrary border between short and long term storage. Beyond $\sim 10^{4}$ turns storage several synchrotron oscillations take place in the bunch and the prediction of the beam evolution becomes more difficult, because the typical space charge effect is coupled with the longitudinal motion. This regime is encountered in the SIS100 synchrotron in the FAIR project at GSI [6]. It is planned that SIS100 [7, 8] will store bunches of $\mathrm{U}^{+28}$ ion beam for a time of the order of a second (few $10^{5}$ turns) with a tuneshift of $\Delta Q \sim-0.3$. The ring nonlinearities in this long term storage of a high intensity bunch play an important role in the beam quality control and beam loss. Protection of cold super-conducting parts of magnets imposes uncontrolled beam loss at the few $\%$ level [9], which is critical for the $\mathrm{U}^{+28}$ research scenario. In fact the large ionization cross section of a $\mathrm{U}^{+28}$ ion beam with residual gas atoms limits the uncontrolled beam loss to $1 \%$ [10]. A beam loss exceeding this threshold triggers a progressive vacuum degradation due to desorption [11], which considerably reduces the beam lifetime [12].

\section{High Intensity Effects for Short Term Storage}

The high intensity in short term storage is responsible for several beam degradation mechanisms. For high intensity it is meant that the space charge tuneshift is not much exceeding the "conventional" value of $\Delta Q \sim-0.25$. A first consequence of the space charge in a beam is that any perturbation to the spatial beam profile causes an oscillatory 2D response of the beam as a whole, which can be decomposed in modes [13]. When the frequency of a mode $\omega=n_{x} Q_{x 0}+n_{y} Q_{y 0}+\Delta \omega$ satisfies the condition $\omega=n_{x} Q_{x 0}+n_{y} Q_{y 0}+\Delta \omega=0$, the corresponding mode can be unstable unstable [14]. Note that self consistent effects in the absence of synchrotron motion can reduce the emittance blow-up for non KV-distributions [15]. A second effect of the high intensity occurs when the force created by the beam resonates with the frequency of particles inside the beam itself creating parametric resonances [16]: a classical example is given by the envelope mode of frequency $\omega=2 Q_{x 0}+\Delta \omega$ which acts on particles in the beam when $2 \omega=N_{s}$, where $N_{s}$ is the superperiodicity. This resonance occurs for a phase advance slightly above $90^{0}$ per period [17]. The beam modes may also grow when driven by lattice resonances $[18,19]$, but even in this case, the response of the beam occurs at a resonance condition, which includes the coherent shift $\Delta \omega$, i.e. $\omega=n$. A different collective response occurs when the nonlinear force created by 
the space charge is modulated by the lattice and creates the so called structure resonances [20]. In presence of lattice gradient errors the perturbed envelope can also excite single particle resonances (non-structure resonances), which might be stronger than the machine resonances of the same order [20].

\section{Single Particle Resonance Condition in Absence of Space Charge}

At low intensity the main causes of particle loss are due to nonlinear resonances. The ring nonlinearities cause a reduction of the domain of stability (dynamic aperture), and the goal of the ring designer is to make this set as large as to contain the beam. The analysis of the properties of the nonlinear orbits can be performed via normal forms, or with a perturbative approach [21]. Single particle resonances $n_{x} Q_{x 0}+n_{y} Q_{y 0}=m$ should be avoided up to a certain order not to have the dynamic aperture shrinking inside the beam. The transverse-longitudinal coupling induces resonance/instabilities, which appear as synchro-betatron resonances with a set of synchrotron sidebands according to the resonance condition $Q_{x, y}=n \pm m Q_{z}$ [22]. This result holds also when lattice resonances are excited as the chromaticity, creating a tune modulation, may push individual particles across a resonance [23].

\section{TRANSVERSE DETUNING FOR A SINGLE CROSSING OF A RESONANCE}

The role of the transverse amplitude dependent tuneshift is beneficial for avoiding emittance growth when the bare tune is not time dependent. In fact, if a ring is tuned on a resonance, as a particle amplitude grows, its tune changes in a dynamical process, which stops when the particle is brought off the resonance. If the detuning follows the dependence $\Delta Q_{a}\left(\epsilon_{x}\right)=a_{1} \epsilon_{x}+a_{2} \epsilon_{x}^{2}+O\left(\epsilon_{x}^{3}\right)$ and the stopband $\Delta Q_{s b}$ is located in $\left[Q_{x, s 1}, \ldots, Q_{x, s 2}\right]$, then the maximum single particle emittance $\epsilon_{x}^{*}$ to bring the particle out of the resonance can be estimated as $\Delta Q_{a}\left(\epsilon_{x}^{*}\right)=Q_{x 0}-Q_{x, s 1}$ if $\partial Q_{a} / \partial \epsilon_{x}<0$. This effect is very strong for high intensity 2D continuous beams [24], but it can be constructed in a ring by choosing properly a set of nonlinear elements [25]. The limiting effect of the amplitude dependent detuning is seen in the phase space by the appearance of stable island.

Completely different is the role of the amplitude dependent detuning when we consider the crossing of a resonance by changing dynamically the machine tune. If the detuning $\Delta Q_{a}$ due to amplitude growth acts against the change of $Q_{x 0}$ and if the crossing is slow enough (with respect to the instantaneous growth rate in the stop-band), the particle will remain on one side of the resonance [26]. The particle amplitude will grow according to $\Delta Q_{a}\left(\epsilon_{x}^{*}\right) \simeq$ $Q_{x, s 2}-Q_{x 0}$, if the bare tune continues to shift away from the stop-band. In case the detuning acts in the same direction of the bare tune variation, if $\partial Q_{a} / \partial \epsilon_{x}$ is large enough, the particle tune will jump through the resonance and the emittance increase will be $\Delta \epsilon_{x} \leq \Delta Q_{s b} /\left|\partial Q_{a} / \partial \epsilon_{x}\right|$; if the detuning gradient is small, as for a particle at the tail of the transverse distribution, then the space charge detuning is not enough and the particle amplitude grows again according to the average residence time in the stop-band. We refer to these two types of crossing as "from above" and "from below" the resonance. In Fig 2 is shown an

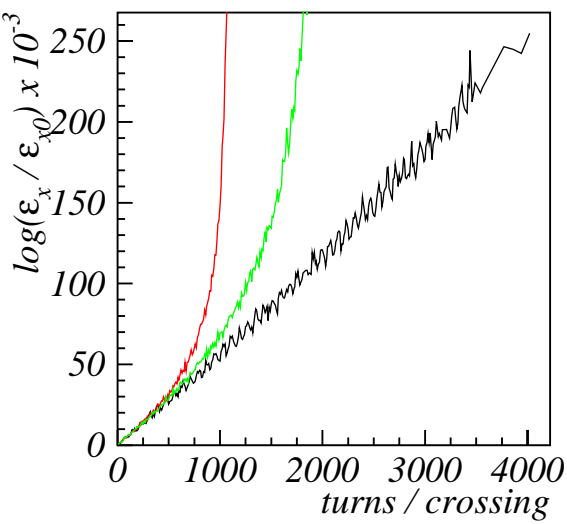

Figure 2: Emittance growth as function of the crossing speed (from above) of a third order resonance without space charge (black curve), with $\Delta Q_{x}=-0.1$ (red curve), with $\Delta Q_{x}=-0.05$ (green curve).

example of emittance increase for a crossing of a third order resonance as function of the crossing speed. The tune interval spanned is \pm 0.15 with respect the center of the resonance. The black curve shows the response of the $2 \mathrm{D}$ beam at low intensity. For a crossing speed smaller than $4 \times 10^{3}$ turns/crossing, the relative growth of the rms emittance is unbounded as the time spent inside the resonance is enough to lose particles. The red curve shows instead the response of a beam with space charge tuneshift of $\Delta Q_{x}=-0.1$. The snowplow effect brings the core particles to larger amplitudes at faster crossing speed: now the beam emittance diverges for crossing speed larger than $10^{3}$ turns/crossing. The green curve shows an intermediate tuneshift for $\Delta Q_{x}=-0.05$. Self consistent studies on single passage resonance crossing are reported in [27].

\section{PARTICLE TRAPPING INTO A RESONANCE}

Alternatively the bare tune can be set close to a resonance, but resonance strength and amplitude dependent detuning are controlled via lattice nonlinear elements [28]. In this case the control on the tune amplitude dependency allows a control on the island position. It is possible to induce island motion, and particularly to move an island of size $\Delta x$ across a particle orbit. According to the island crossing speed $\delta x_{f} / \partial n$ through the particle orbit characterized by $x_{\text {max }}^{\prime}$, there are two possible scenarios: 1) The particle remains trapped into the island and follows its fixed points; 2) The island is moving too fast and the particle receives an orbit jump. When the island crossing satisfies an adiabaticity criteria: $x_{\max }^{\prime} \gg \partial x_{f} / \partial n$ [28], or alternatively 
$\left(\partial x_{f} / \partial n\right) /\left(\Delta x Q_{x f}\right) \ll 1[29]$, particles inside the islands remain trapped. Here $Q_{x f}$ is the tune of the fixed points. The general study of the crossing of the separatrix can be found in [30, 31]. In Fig. 3a,b we show this effect for a tune $Q_{x 0}$ set at 0.03 above the third order resonance. The space charge is progressively increased in 5000 turns from $\Delta Q_{x}=0$ to $\Delta Q_{x}=-0.1$. Note in Fig. 3b the characteristic orbit of a particle trapped, which follows the third order island. The particle reaches a maximum amplitude
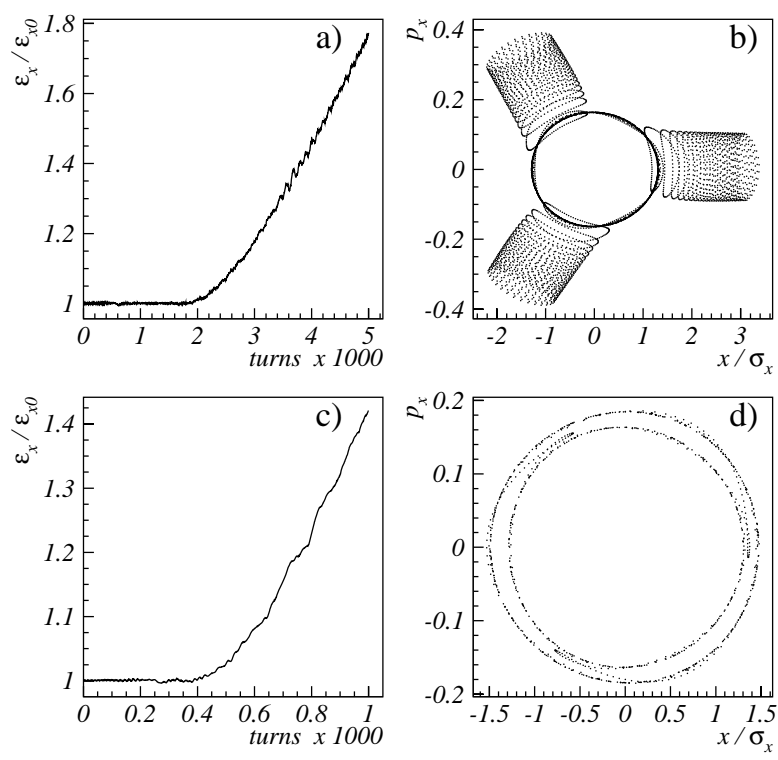

Figure 3: Rms emittance evolution as function of number of turns to increase $\Delta Q_{x}$; The pictures a),b) are an increase in 5000 turns, while in c),d) a faster crossing in 1000 turns is shown.

of $x=3.37 \sigma_{x}$ starting from $x=1.3 \sigma_{x}$. In Figs. $3 \mathrm{c}, \mathrm{d}$ are shown similar results when the maximum tune-spread is reached instead in 1000 turns. Now the crossing of the resonance is too fast and the stable island cannot trap the test particles. As the position of the island is controlled by space charge $\Delta Q_{x}$, trapped particles reach further distances in phase space for higher beam intensities. For example repeating the slower crossing for $\Delta Q_{x}=-0.2 \mathrm{we}$ find that the test particle extends in $x$ up to $5.11 \sigma_{x}$.

\section{HIGH INTENSITY BUNCH DYNAMICS IN PROXIMITY OF A RESONANCE}

When we consider the storage of a high intensity bunch all the elements of the previous discussion have to be taken into account. For a fixed $Q_{x 0}$, the synchrotron motion makes each particle move along the bunch experiencing a modulated space charge at twice the synchrotron oscillation frequency. When the particle is at $z=0$, the tunespread for $x \sim y \sim 0$ is the largest, $\Delta Q_{x}(0)$; when it reaches its maximum longitudinal amplitude $z_{\max }$ it experiences a reduced tune-spread $\Delta Q_{x}\left(z_{\max }\right)$. For bunches with small aspect ratio $\sigma_{x} / \sigma_{z}$, which is typical for bunches in a ring, the transverse electric field is obtained from the local coasting beam approximation: the transverse electric field can be computed with the local current assuming a coasting beam. For $\sigma_{x} / \sigma_{z}=3 \times 10^{-4}$ a relative error better than $1 \%$ with respect to the real $3 \mathrm{D}$ electric field is obtained. The dependence of the tune-spread from the longitudinal position of a particle is well approximated by $\Delta Q(z)=\Delta Q_{x} \exp \left[-0.5\left(z / \sigma_{z}\right)^{2}\right]$ for a longitudinal Gaussian distribution. If the bare tune $Q_{x 0}$ is placed above a resonance, then the islands will be created and their fixed point will be a function of $z$ and of the maximum space charge $\Delta Q_{x}$. Consequently a particle in a bunch will be periodically crossed by islands. Note that the dynamics involved in the trapping is more complex than a repulsive snowplow effect of a single passage. In fact, if in one half synchrotron oscillation islands move out, then in the next half they move in creating a periodic migration.

\section{Difference with Synchro-betatron Resonances}

In the synchro-betatron resonances the lack of a strong amplitude dependent detuning allows to consider the longitudinal motion as an extra source of harmonics. The single particle tune can be written as $Q_{x}=$ $Q_{x 0}+\Delta Q_{a}\left(\epsilon_{x}\right)+\delta Q_{x, c h r}(s)$ with $\delta Q_{x, c h r}(s)=$ $\sum_{m=1} \xi_{m} \cos \left[m\left(Q_{z 0} / R\right) s\right]$, hence the appearance of sidebands in the resonance condition $Q_{x, y}=n \pm m Q_{z}$. If the detuning $\Delta Q_{a}$ is imposed externally by lattice nonlinearities modulated with wavelength $L / Q_{e}$, where $L$ is the length of the ring, then the external tune $Q_{e}$ will be brought into the resonance relation. However, in the case of a high intensity bunch the space charge creates the single particle tune as

$$
Q=Q_{x 0}+\frac{\Delta Q_{x} e^{-\left(\frac{z_{0}}{2 \sigma_{z}}\right)^{2} \cos \left[\left(\frac{Q_{z 0}}{R}\right) s\right]^{2}}}{1+\left[\tilde{x} /\left(2 \sigma_{x}\right)\right]^{2}}+\delta Q_{x, c h r}(s) .
$$

for a particle in the linear part of the bucket. Here $\tilde{x}$ is the maximum transverse amplitude. The space charge detuning [29] cannot be treated as a normal lattice nonlinearity: firstly the space charge force is continuously distributed along the ring and is modulated with the lattice optics creating structure driving terms. Secondly, by expanding in $\tilde{x}$ we find $1 /\left\{1+\left[\tilde{x} /\left(2 \sigma_{x}\right)\right]^{2}\right\}=\sum_{n=0}(-1)^{n}\left[\tilde{x} /\left(2 \sigma_{x}\right)\right]^{2 n}$, which indicates that all these components should be simultaneously accounted for particles with amplitude of the order of $\sigma_{x}$. This prevents the contribution of a single harmonic to the synchro-betatron sideband. Only if $\left|\Delta Q_{x}\right|<Q_{z 0}^{-1}$ sidebands can appear. Due to this property of the space charge term, the effect of the chromaticity in a high intensity bunch should be revised as well.

\section{Trapping and Scattering Regime}

In a resonance periodic crossing regime particles have a finite probability of being trapped or scattered by islands. In Fig. 4 is shown an example of the dependence of a beam evolution during a storage time of 100 synchrotron oscillations. We take the SIS18 with $Q_{x 0}=4.3433$ and $Q_{y 0}=3.29$, where we add a sextupole to a linear constant focusing lattice to excite the resonance $3 Q_{x 0}=13$. 
The space charge gives $\Delta Q_{x}=-0.1$, and the transverse beam sizes are equal. In Fig. 4a we show the emittance growth for varying synchrotron tune (expressed in turns for one synchrotron oscillation as $N_{z 0}$ ). We see that the emittance growth increases, but above $N_{z 0}=500$ it remains constant to $\sim 3.5$. This means that in 100 synchrotron oscillations all particles crossing the resonance fill the region spanned by the islands. The black curve, counting the particles beyond $3 \sigma_{x}$ saturates at $\sim 15 \%$. which indicates that the process of diffusion is fast enough with respect to the storage time. In Fig. $4 \mathrm{~b}$ we have calculated the single particle emittance for one particle of this bunch with
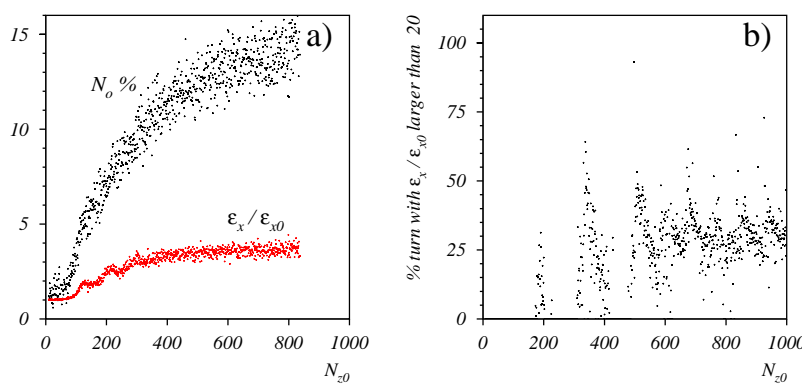

Figure 4: a) Rms emittance growth (red) and $\%$ of particle out of $3 \sigma_{x}$ (black); b) Residence of test particle out of the beam as function of $N_{z 0}$.

$x=1.5 \sigma_{x}, p_{x}=y=p_{y}=p_{z}=0$, and $z=3 \sigma_{z}$ and show the $\%$ of stored turns where $\epsilon_{x} / \epsilon_{x 0}>20$. This result is shown as function of $N_{z 0}$, which shows the existence of a scattering regime where this particle is not brought to large amplitude (for $N_{z 0}<180$ ). When the synchrotron motion is sufficiently slow, in our example for $N_{z 0}>180$, the island will always cross the particle orbit in an adiabatic regime and trapping may occur accelerating the diffusion process.

\section{Role of Chromaticity in High Intensity Beams}

Including chromaticity complicates the particle dynamics. The space charge driven tune modulation periodicity, is half of the tune modulation introduced by the chromaticity, which equals the synchrotron motion periodicity. To understand the behaviour of beams in presence of the chromaticity we consider the particle tune $Q_{x}=Q_{x 0}+\delta Q_{x, c h r}$ including the chromaticity induced tune shift. If $Q_{x}$ gets close to the resonance from above, the trapped particles will be brought to large amplitude. Therefore if $\Delta Q_{x, c h r}$ is the tune-spread induced by the chromaticity, setting the bare tune in the region $Q_{x, \text { res }}<Q_{x 0}<Q_{x, \text { res }}+\Delta Q_{x, \text { chr }}$ will always allow some particles in the bunch to hit the pipe and be lost. A more detailed description of this beam loss mechanism is found in [29].

\section{THE ELEMENTS OF BEAM LOSS PREDICTION}

The key ingredients, which determine the long term beam loss in a high intensity bunch are: 1) Distance from the resonance $\left.Q_{x 0}-Q_{x, \text { res }} ; 2\right)$ Space charge tune-spread $\left|\Delta Q_{x}\right|$; 3) Chromaticity tune-spread $\left|\Delta Q_{x, c h r}\right|$, here assumed smaller then $\left|\Delta Q_{x}\right|$;4) Resonance strength and $Q_{z 0}$. When $\left|\Delta Q_{x}\right|>Q_{x 0}-Q_{x, \text { res }}>0$, a resonance crossing regime takes place. The outer transverse position of the fixed points occurs at $z=0$, and depends on $Q_{x 0}-Q_{x, \text { res }}$ : for $Q_{x 0} \rightarrow Q_{x, \text { res }}$ their position is (virtually) infinite; The inner transverse position of the fixed points is at $x=0$ in two symmetric longitudinal positions, which can be identified by a longitudinal single particle emittance $\epsilon_{z_{t}}$. If a particle has $\epsilon_{z}<\epsilon_{z_{t}}$ then the resonance is never crossed, whereas if $\epsilon_{z}>\epsilon_{z_{t}}$ it crosses 4 times per synchrotron oscillation. The total number of particles, which periodically cross the resonance, is estimated for a 3D Gaussian distribution as $\Delta N / N=\alpha\left(Q_{x 0}-Q_{x, \text { res }}\right) /\left|\Delta Q_{x}\right|$. In this formula valid for $0<\Delta N / N<1, \alpha$ depends on the topology of the islands; its lower limit is obtained by a direct integration, which yields $\alpha>1$. If the outer position of islands intercepts the beam pipe or reaches the dynamic aperture, particles with $\epsilon_{z}>\epsilon_{z_{t}}$ are lost consistently with $Q_{x 0}-Q_{x, \text { res }}$ causing a longitudinal bunch shortening (see [32]). The presence of the chromaticity creates a beam loss stop-band right above the resonance as large as $\left|\Delta Q_{x, c h r}\right|$. The beam loss rate, depends on the resonance strength and $Q_{z 0}$ (or $N_{z 0}$ ) which determine the number of particles in the adiabatic crossing regime. A code-code benchmarking on the trapping effect can be found in [32].

As example we apply the MICROMAP library with frozen space charge to the SIS100 for the working point $Q_{x 0}=18.84, Q_{y 0}=18.73$ [7]. The space charge tuneshift is $\Delta Q_{x}=-0.14, \Delta Q_{y}=-0.25$, for a 3D Gaussian distribution with rms emittances $\epsilon_{x}=8.75 \mathrm{~mm}-\mathrm{mrad}, \epsilon_{y}=3.5$ $\mathrm{mm}$-mrad. The bunch has $0.75 \times 10^{11} \mathrm{U}^{+28}$ ions. In Fig. 5a is shown a tune scan of the short term dynamic aperture computed in 1000 turns. For the selected working point the dynamic aperture is $\sim 3.5 \sigma$. Note the presence of two weak high order systematic resonances $2 Q_{x}+8 Q_{y}=186$, and $7 Q_{x}+Q_{y}=150$, which cross the the space charge tune-spread diamond. However, given their high order, the size of islands created is small setting the SIS100 in a scattering regime. The relatively large beam size with respect to the dynamic aperture creates a highly nonlinear regime where island are close to the boundary of stability and particles in the tail are lost. The beam loss developing in $10^{5}$ turns is found $\sim 5 \%$ on a bunch of 2000 test macroparticles. The first $\sim 2.5 \%$ beam loss quickly occurs at the beginning for the particles in the tail as beyond the mechanical acceptance. As the dynamic aperture is close the beam tail and mechanical apertures, we cannot evaluate the impact of the scattering regime in the beam loss shown in Fig. 5 which are affected by non-regular motion. Further studies with larger number of macroparticles and several beam distribution are in progress.

\section{Experimental Verification}

An experimental campaign to investigate these phenomena took place at the CERN-PS in 2002-2003 [33]. A bunched 

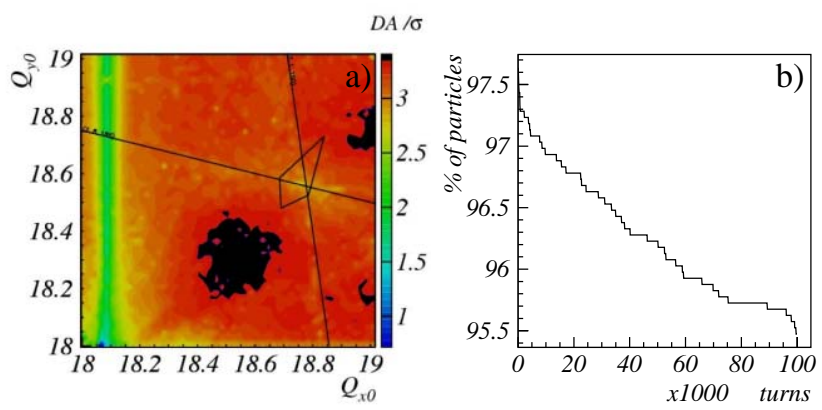

Figure 5: a) Tune diagram with SIS100 WP1 working point; b) Beam loss for a Gaussian beam.

beam with $\Delta Q_{x}=0.075$ was stored for $5 \times 10^{5}$ turns and $Q_{y 0}=6.12$ for several values of $Q_{x 0}$. In this table are reported the main results (with index $s$ the results of simulations)

\begin{tabular}{|c|c|c|c|}
\hline$Q_{x 0}$ & 6.25 & 6.265 & 6.28 \\
\hline$\Delta \epsilon_{x} / \epsilon_{x}$ & $-14 \%$ & $-37 \%$ & $42 \%$ \\
\hline$\left(\Delta \epsilon_{x} / \epsilon_{x}\right)_{s}$ & $-10 \%$ & $30 \%$ & $87 \%$ \\
\hline$\Delta N / N$ & $-17 \%$ & $-32 \%$ & $-9 \%$ \\
\hline$(\Delta N / N)_{s}$ & $-17 \%$ & $-16 \%$ & $0 \%$ \\
\hline
\end{tabular}

The beam loss regime coincides with the chromaticity tunespread of $\Delta Q_{c h r} .=0.028$ for the rms momentum spread of $\Delta p / p=1.5 \times 10^{-3}$ and the PS natural chromaticity. A simulation including the chromaticity yields a maximum beam loss of $16 \%$ at $Q_{x 0}=6.265$ which is half the measured loss. The large emittance obtained in the simulations for $Q_{x 0}=6.265,6.28$ is consistent with a reduced beam loss. In a previous simulation ignoring the chromaticity we have found only $8 \%$ loss [32]. The still remaining discrepancy will be subject of future studies, which should include an estimate of the effect of the fully self-consistent space charge (2D $\frac{1}{2}$ see [34]).

\section{Outlook}

Experimental studies on trapping effects in high intensity beams are in progress at GSI with an official dedicated beam time (S317 experiment). The evaluation of data from these experiments will be part of a future report. The impact of the high intensity nonlinear beam dynamics on the SIS100 halo collimation system will be evaluated in the near future. The principles of the trapping mechanisms driven by high intensity beams are also found in incoherent electron clouds [35]. A present benchmarking effort in this area of studies is in progress [36].

Funded by EU design study (contract 515873DIRACsecondary-Beams).

The author is thankful to L. Ahrens, G. Arduini, W. Fischer, I. Hofmann and P. Zenkevich for useful discussions.

\section{REFERENCES}

[1] S. Henderson et al., Proc. of EPAC 2006, p. 345, MOPCH129.

05 Beam Dynamics and Electromagnetic Fields
[2] L. Ahrens et al., Proc. of PAC 1999, p. 614, FRAR3.

[3] G. Arduini, private communication.

[4] P. Spiller et al., Proc. of PAC 2005, p. 294, FOPA003.

[5] F. Noda et al., Proc. of PAC 2005, p. 2759, MPPE043.

[6] D. Kraemer, THXAB02, these proceedings.

[7] P. Spiller, TUPAN014, these proceedings; G. Franchetti et al., Proc. of EPAC 2006 p.2793, THPCH005.

[8] SIS100 Technical report, http://www.gsi.de/fair/reports/btr.html.

[9] E. Mustafin et al., Proc. of EPAC 2004, p. 1408, TUPLT112; E. Mustafin et al., Proc. of PAC 2005, p. 3943, FPAE075.

[10] C. Omet, TUPAN013, these proceedings.

[11] H. Kollmus et al., Proc. of EPAC 2006, p. 1426, TUPCH174; A.W. Molvik et al., Phys. Rev. Lett. 98054801 (2006).

[12] C. Omet, et al., New J. Phys. 8, No 11, (2006) 284.

[13] I. Hofmann, Phys. Rev. E 57, 4713 (1998).

[14] I. Hofmann et al., Phys. Rev. ST Accel. Beams 6, 024202 (2003).

[15] I. Hofmann, G. Franchetti, A.V. Fedotov, Proc. of 20th ICFA worksop, AIP 642, 248 (2002).

[16] A.V. Fedotov, Proc. of PAC 2003, p. 383, WOAB002.

[17] J. Struckmeier and M. Reiser, Part. Accel. 14, p. 227 (1984); S. Cousineau et al., Phys. Rev. ST Accel. Beams 6094202 (2003).

[18] M. Venturini and R.L. Gluckstern, Phys. Rev. ST Accel. Beams 3, 034203 (2000).

[19] M. Aslaninejad and I. Hofmann, Phys. Rev. ST Accel. Beams 3, 034203 (2000).

[20] S. Machida, NIM A 309, p. 43, (1991); ibid. 384, p. 316, (1997).

[21] A. Bazzani et al., CERN-94-02, (1994); G. Guignard, CERN 78-11, (1978).

[22] A. Piwinski and A. Wrulich, DESY 76/07 (1976).

[23] S.R. Mane and W.T. Weng, NIM A, 306, 9, (1991).

[24] A.V. Fedotov and I. Hofmann, Phys. Rev. ST Accel. Beams 5, 024202 (2002).

[25] R. Cappi and M. Giovannozzi, Phys. Rev. Lett. 88, 104801 (2002).

[26] G. Franchetti, I. Hofmann, M. Aslaninejad, Phys. Rev. Lett. 94, 194801 (2005).

[27] I. Hofmann and Franchetti, THPAN017, these procedings.

[28] A. W. Chao and M. Month, NIM 121, 129-138 (1974).

[29] G. Franchetti and I. Hofmann, NIM A 561, 195, (2006).

[30] A. Schoch, CERN Report, CERN 57-23, (1958).

[31] A.I. Neishtadt, Sov. J. Plasma Phys. 12, 568-573 (1986).

[32] G. Franchetti et al., Proc. of 33rd ICFA worksop, AIP 773, 137 and (2004); G. Franchetti et al., Proc. of 39rd ICFA worksop (2006), p. 344, THBW01.

[33] G. Franchetti et al., Phys. Rev. ST Accel. Beams 6, 124201 (2003); E. Metral et al., NIM A, 561,257,(2006).

[34] C. Benedetti et al., THPAN030, these proceedings.

[35] E. Benedetto et al., Phys. Rev. Lett. 97, 034801 (2006); K. Ohmi and K. Oide, Phys. Rev. ST Accel. Beams 10, 014401 (2007).

[36] F. Zimmermann et al., THPAN075, these proceedings. 